Internat. J. Math. \& Math. Sci.

Vol. 24, No. 9 (2000) 595-612

S0161171200004403

(C) Hindawi Publishing Corp.

\title{
FIXED POINTS AND SELECTIONS OF SET-VALUED MAPS ON SPACES WITH CONVEXITY
}

\author{
PETER SAVELIEV
}

(Received 22 November 1999)

\begin{abstract}
We provide theorems extending both Kakutani and Browder fixed points theorems for multivalued maps on topological vector spaces, as well as some selection theorems. For this purpose we introduce convex structures more general than those of locally convex and non-locally convex topological vector spaces or generalized convexity structures due to Michael, van de Vel, and Horvath.
\end{abstract}

Keywords and phrases. Fixed point, continuous selection, generalized convexity.

2000 Mathematics Subject Classification. Primary 47H04, 47H10, 52A01, 54C65, 54H25.

1. Introduction. The origin of our fixed point theorem is the following two classical results due to Fan, Glicksberg, and Kakutani [7, 8, 19] and Browder [3], respectively (see also [31]).

THEOREM 1.1 (Kakutani fixed point theorem). Let $X$ be a nonempty convex compact subset of a locally convex Hausdorff topological vector space, and let $F: X \rightarrow X$ be a u.s.c. multifunction with nonempty convex closed images. Then $F$ has a fixed point.

THEOREM 1.2 (Browder fixed point theorem). Let $X$ be a nonempty convex compact subset of a Hausdorff topological vector space, and let $G: X \rightarrow X$ be a multifunction with nonempty convex images and preimages relatively open in $X$. Then $G$ has a fixed point.

Similarly, our selection theorem unites the following two results due to Michael [20] and Browder [3] (see also [31]).

THEOREM 1.3 (Michael selection theorem). Let $X$ be a paracompact Hausdorff topological space, and let $Y$ be a Banach space. Let $T: X \rightarrow Y$ be an l.s.c. multifunction having nonempty closed convex images. Then $T$ has a continuous selection.

THEOREM 1.4 (Browder selection theorem). Let $X$ be a paracompact Hausdorff topological space, and let $Z$ be any topological vector space. Let $T: X \rightarrow Z$ be a multifunction having nonempty convex images and open preimages. Then $T$ has a continuous selection.

The main goal of this paper is to provide a uniform approach to these four results. In recent years, these theorems have been generalized in numerous ways $[1,2,4,12$, $17,23,24,28,29]$. Our main theorems contain as immediate corollaries a number of these results; however, we do not attempt to include all of them.

Our approach is based on Michael's [20] and Browder's techniques [3] and the study of abstract convexity structures on topological spaces originated in works of 
Michael [21], van de Vel [30], Horvath [15], and others (see Section 12). Given a topological (or uniform) space $Y$, van de Vel introduces the class of "convex" sets as a class of subsets of $Y$ closed under intersections. Horvath defines "convex hulls" of finite subsets of $Y$. Michael, on the other hand, considers an analogue of convex combination functions of vector spaces: $k_{n}\left(d_{0}, \ldots, d_{n}, a_{0}, \ldots, a_{n}\right)=\sum_{i=0}^{n} d_{i} a_{i}$, where $\left(d_{0}, \ldots, d_{n}\right)$ is an element of the $n$-simplex $\Delta_{n}$, for certain combinations $\left(a_{0}, \ldots, a_{n}\right)$ of elements of the space. For topological vector spaces, convex combination functions are continuous with respect to $d_{0}, \ldots, d_{n}$. But for locally convex topological vector spaces, these functions are continuous with respect to $a_{0}, \ldots, a_{n}$ as well.

We follow Michael's approach (Definition 1.5) because, as we will see, it is especially convenient for selection and fixed point problems. For the former, $Y$ does not have to be "convex." Following Michael, we avoid this situation, while in van de Vel's and Horvath's constructions there is the largest "convex" set. Our construction extends Michael's definition in such a way that most of van de Vel's and Horvath's fixed point and selection results are included.

Assuming that $\mathscr{B}$ is a base of the metric uniformity of a space $Y$, we state Michael's definition in a form equivalent to the original if $Y$ is compact.

DEFINITION 1.5. A sequence of pairs $\left\{\left(M_{n}, k_{n}\right)\right\}$ is a Michael's convex structure if for all $n \geq 0, M_{n} \subset Y^{n+1}, k_{n}: \Delta_{n} \times M_{n} \rightarrow Y$ ( $M_{n}$ can be empty) and the following is satisfied:

(a) if $x \in M_{0}$, then $k_{0}(1, x)=x$,

(b) if $x \in M_{n}, n \geq 1, i \leq n$, then $\partial_{i} x \in M_{n-1}$, and if $t_{i}=0$ for $t \in \Delta_{n}$, then $k_{n}(t, x)=$ $k_{n-1}\left(\partial_{i} t, \partial_{i} x\right)$, where $\partial_{i}$ is the operator that omits the $i$ th coordinate,

(c) if $x \in M_{n}, n>i \geq 0$, and $x_{i}=x_{i+1}$, then for $t \in \Delta_{n}$,

$$
k_{n}(t, x)=k_{n-1}\left(t_{1}, \ldots, t_{i-1}, t_{i}+t_{i+1}, t_{i+2}, \ldots, t_{n}, \partial_{i} x\right),
$$

(d) for each $x \in M_{n}$, the map $k_{n}(\cdot, x)$ is continuous,

(e) for any $U \in \mathscr{B}$ there is a $W \in \mathscr{B}$ such that for all $n \geq 0, t \in \Delta_{n}, x=\left(x_{0}, \ldots, x_{n}\right)$, $y=\left(y_{0}, \ldots, y_{n}\right) \in M_{n}$, we have

$$
\left(x_{i}, y_{i}\right) \in W, \quad 0 \leq i \leq n, \Longrightarrow\left(k_{n}(t, x), k_{n}(t, y)\right) \in U .
$$

We relax Michael's conditions in several ways (Section 2). First, we do not assume that $Y$ is metrizable but only uniform. Second, we allow the convex combination function to be multivalued. Third, instead of a sequence of maps $\left\{k_{n}\right\}_{n=1}^{\infty}$ connected by conditions (b) and (c), we use a sole multifunction $C$ from a subset of the set $\Delta(Y)$ of all formal convex combinations of elements of $Y$ into $Y$, which makes it easier to prove existence of $C$. We consider a certain convexity multifunction $C$ satisfying continuity conditions (D) and (E) similar to conditions (d) and (e) above. Condition (E) allow us to carry out most of the selection and fixed point constructions (Sections 7 and 10) and only as the last step do we consider various continuity conditions with respect to $t$ (condition (D)), which ensures continuity of selections and existence of fixed points of continuous maps. The conditions of Michael's definition do not hold for non-locally convex topological vector spaces and we have to deal with them in order to obtain Theorems 1.2 and 1.4. To resolve this problem, we introduce a second topology $Z$ on $Y$. 
As a result, the convexity satisfies the two continuity requirements, but with respect to two (possibly different) topologies. Consequently, by choosing an appropriate topological structure on $Y$, we are able to obtain Theorems 1.1 (for $Z=Y$ ) and 1.2 (for $Y$ discrete) as immediate corollaries of our fixed point result (Theorem 10.2). In the same manner we derive Theorems 1.3 and 1.4 from our selection theorem (Theorem 7.4).

We also use the fact that the presence of convex combinations reduces the question of existence of fixed points for a certain class of multifunctions on topological spaces to the question of existence of fixed points of multifunctions on simplexes (see [2, 24]). This enables us to use the Brouwer fixed point theorem for $\Delta_{n}$, or, more generally, the theorem below that is contained in Corollary 2.3 of Gorniewicz [9] (for a stronger result, see [26]). A multivalued map $F: X \rightarrow Y$ is called admissible in the sense of Gorniewicz if it is closed valued u.s.c. and there exist a topological space $Z$ and two single-valued continuous maps $p: Z \rightarrow X, q: Z \rightarrow Y$ such that $p$ is proper and for any $x \in X$, (i) $p^{-1}(x)$ is acyclic, and (ii) $q\left(p^{-1}(x)\right) \subset F(x)$. Many fixed point theorems in the existing literature will be shown to be reducible to this theorem.

THEOREM 1.6. Any admissible map in the sense of Gorniewicz and, in particular, any composition of acyclic multifunctions, $F: \Delta_{n} \rightarrow \Delta_{n}$ has a fixed point.

Another purpose of this paper is to obtain fixed point theorems for topological spaces without linear or convex structure. To achieve this goal we need to show that a given topological space can be equipped with a convexity structure; that is, to prove existence of convex combination functions. As a corollary we obtain a generalization of the following theorem due to Eilenberg and Montgomery [5].

THEOREM 1.7 (Eilenberg-Montgomery fixed point theorem). Let $X$ be an acyclic compact ANR, and let $F: X \rightarrow X$ be an acyclic multifunction. Then $F$ has a fixed point.

2. Convexity on uniform spaces. Throughout the paper, we fix an infinite cardinal number $\omega$ and an index set $I$ with $|I|=\omega$. We assume that $\omega$ is large enough in the sense that $\omega \geq 2^{|X|}$ for each space $X$ involved. Let $\Delta_{\omega}$ be the infinite dimensional simplex spanned by the unit vectors $e_{k}, k \in I$, of $[0,1]^{\omega}$. For any nonempty subset $K$ of $I$, let $\Delta_{K}$ denote the convex hull of the set $\left\{e_{k}: k \in K\right\}$ in $\Delta_{\omega}$ (i.e., $\Delta_{K}=\{d=$ $\left.\left.\left(d_{i}\right)_{i \in I} \in \Delta_{\omega}: i \notin K \Rightarrow d_{i}=0\right\}\right)$ and let $\Delta_{n}$ be any $n$-simplex in $\Delta_{\omega}$ spanned by some unit vectors. We let $\Delta(Y)=\Delta_{\omega} \times Y^{\omega}$ and assume the following.

Convention. If $(d, a) \in \Delta(Y)$, then $d_{i} \in[0,1], a_{i} \in Y, i \in I$, are the coordinates of $d \in[0,1]^{\omega}, a \in Y^{\omega}$.

For any $A \subset Y$, we define the set of all formal convex combinations of elements of $A$ :

$$
\Delta(A)=\left\{(d, a) \in \Delta(Y): d_{i} \neq 0 \Longrightarrow a_{i} \in A, i \in I\right\} .
$$

The following are also fixed:

(c1) $Y$ is a uniform space with a minimal (uniform) open base $\mathscr{B}$ (i.e., one with the smallest cardinality), partially ordered by inclusion,

(c2) $Z$ is a topological space on $Y$ (the topology of $Z$ is not necessarily the uniform topology of $Y$ ),

(c3) $\mathscr{V}$ is a class of multifunctions that will be specified later, 
(c4) $\mathscr{A} \subset 2^{Y} \backslash\{\varnothing\}$ is a class of subsets of $Y$ called admissible sets ( $A$ may be empty),

(c5) conv: $\mathscr{A} \rightarrow 2^{Y} \backslash\{\varnothing\}$ is a function, and $\operatorname{conv}(A)$ is called the convex hull of $A \in \mathscr{A}$,

(c6) $\mathscr{C}=\{A \in \mathscr{A}: \operatorname{conv}(A) \subset A\} \cup\{\varnothing\}$ is the set of convex subsets of $Y$,

(c7) $Q=\cup_{A \in \mathscr{A}} \Delta(A), Q^{\prime}$ is a subset of $\Delta(Y)$ containing $\cup_{A \in \mathscr{A}, W \in \mathscr{B}} \Delta(B(A, W)$ ),

(c8) $C: Q^{\prime} \rightarrow Y$ is a multifunction called convex combination.

DefinITION 2.1 (main definition). The triple $\kappa=(Y, C, Z)$ is called a convexity associated with $\mathscr{A}$, conv, $\mathscr{V}, Q^{\prime}$ (this part will often be suppressed) if the following conditions are satisfied:

(D) for any $a \in Y^{\omega}$, if $\Delta_{n} \times\{a\} \subset Q^{\prime}, n \geq 0$, then the multifunction $C(\cdot, a): \Delta_{n} \rightarrow Z$ belongs to $\mathscr{V}$,

(E) for any $U \in \mathscr{B}$, there exists $W \in \mathscr{B}$ such that

$$
C(\Delta(B(A, W))) \subset B(\operatorname{conv}(A), U) \text { for all admissible } A \subset Y \text {. }
$$

If $Q^{\prime}=\Delta(Y)$, then the convexity is called global.

Proposition 2.2. If $(Y, C, Z)$ is a convexity, then the following condition is satisfied:

$(\gamma) C(\Delta(A)) \subset \overline{\operatorname{conv}}(A)$, the closure of $\operatorname{conv}(A)$ in $Y$, for all admissible $A \subset Y$.

3. A strong convexity. Let $F_{i}: X \rightarrow Y, i \in J$, be multifunctions, where $J$ is a directed set. Then we say that $\left\{F_{i}: i \in J\right\}$ converges uniformly on $N \subset X$ to a multifunction $F: N \rightarrow Y$ if, for any $U \in \mathscr{B}$, there exists an $i_{0} \in A$ such that

$$
F_{i}(x) \subset B(F(x), U) \quad \forall x \in N, i \in J, i>i_{0} .
$$

Let $\Omega$ denote the set of all finite subsets of the index set $I$. For a fixed $d \in \Delta_{\omega}$, we define elements of the product uniformity of $\{d\} \times Y^{\omega}$ as follows: for any $W \in \mathscr{B}$, $m \in \Omega$, we let

$$
\begin{gathered}
W^{m}=\left\{\left((d, a),\left(d, a^{\prime}\right)\right) \in \Delta(Y) \times \Delta(Y):\left(a_{j}, a_{j}^{\prime}\right) \in W, j \in m\right\}, \\
B^{*}\left((d, a), W^{m}\right)=\left\{\left(d, a^{\prime}\right) \in \Delta(Y):\left(a_{j}, a_{j}^{\prime}\right) \in W, j \in m\right\}, \\
B^{*}\left(S, W^{m}\right)=\cup_{s \in S} B^{*}\left(s, W^{m}\right),
\end{gathered}
$$

where $(d, a) \in \Delta(Y), S \subset \Delta(Y)$.

Consider the following conditions on the objects defined in (c1)-(c9) that loosely correspond to conditions (b)-(e) of Michael's Definition 1.5:

( $\beta$ ) (permutations) if $(d, a),\left(d^{\prime}, a^{\prime}\right) \in Q$ and $\sum_{a_{i}=y} d_{i}=\sum_{a_{i}^{\prime}=y} d_{i}^{\prime}$ for any $y \in Y$, then $C(d, a)=C\left(d^{\prime}, a^{\prime}\right)$,

( $\gamma$ ) (convex hull) $C(\Delta(A)) \subset \overline{\operatorname{conv}}(A)$, the closure in $Y$, for all admissible $A \subset Y$,

( $\delta$ ) (d-continuity) for any $a \in Y^{\omega}$, if $\Delta_{n} \times\{a\} \subset Q^{\prime}, n \geq 0$, then the multifunction $C(\cdot, a): \Delta_{n} \rightarrow Z$ belongs to $\mathscr{V}$ (which is condition (D) of Definition 2.1).

( $\varepsilon$ ) (a-continuity) for any $U \in \mathscr{B}$, there exist $W \in \mathscr{B}, m \in \Omega$, such that

$$
C\left(B^{*}\left((d, a), W^{m}\right)\right) \subset B(C(d, a), U) \quad \forall(d, a) \in Q .
$$


Definition 3.1. The triple $\kappa=(Y, C, Z)$ is called a strong convexity if conditions $(\beta),(\gamma),(\delta)$, and $(\varepsilon)$ are satisfied.

As a direct consequence of the definitions above, we obtain the following for a strong convexity.

LEMmA 3.2. For any $A \subset Y, W \in \mathscr{B}, m \in \Omega$, we have

$$
\Delta(B(A, W)) \subset B^{*}\left(\Delta(A), W^{m}\right) .
$$

THEOREM 3.3. Conditions $(\alpha),(\gamma)$, and $(\varepsilon)$ imply condition (E), so any strong convexity is a convexity.

Proof. Let $U \in \mathscr{B}$ be fixed, and let $U^{\prime} \in \mathscr{B}$ satisfy $4 U^{\prime} \subset U$. By $(\varepsilon)$, there exist $W \in \mathscr{B}, m \in \Omega$, such that

$$
C\left(B^{*}\left((d, a), W^{m}\right)\right) \subset B\left(C(d, a), U^{\prime}\right) \quad \forall(d, a) \in Q .
$$

If $A$ is an admissible set, then $\Delta(A) \subset Q$, so this inclusion holds for all $(d, a) \in \Delta(A)$. Hence

$$
C\left(B^{*}\left(\Delta(A), W^{m}\right)\right) \subset B\left(C(\Delta(A)), 2 U^{\prime}\right) .
$$

Applying consecutively Lemma 3.2, the above inclusion, and condition $(\gamma)$, we obtain condition (E).

\section{Convexity of topological vector spaces}

Definition 4.1. Let $\mathscr{V}$ be the class of all single-valued continuous maps. Then we say that the convexity is continuous.

Some examples of spaces with continuous convexity are listed in this and the following sections.

DefinITION 4.2. A continuous convexity $\kappa=(Y, C, Y)$ (here the topological structures of $Y$ and $Z$ coincide) associated with $\mathscr{A}$, conv, is called regular if it is global and for any $y \in Y$, we have $\{y\} \in \mathscr{A}$ and $\operatorname{conv}(\{y\})=\{y\}$.

Proposition 4.3. Let $Y$ be a convex subset of a locally convex topological vector space. Then $(Y, C, Y)$ with $C$ given by

$$
C(d, a)=\sum_{i} d_{i} a_{i}, \quad(d, a) \in \Delta(Y),
$$

is a regular (strong) convexity associated with $\mathscr{A}=2^{Y}, \operatorname{conv}(A)=\operatorname{co}(A), A \subset Y$ (where $\operatorname{co}(A)$ is the usual convex hull in a vector space).

If the topological vector space is not locally convex Proposition 4.3 fails, which motivates the next definition.

DEFinition 4.4. A continuous convexity $(Y, C, Z)$ is called discrete if $Y$ is discrete (then condition (E) turns into $C(\Delta(A)) \subset \operatorname{conv}(A)$ for all $A \in A$ ). 
Proposition 4.5. Let $Z$ be a convex subset of a topological vector space. Then $(Y, C, Z)$ ( $Y$ is discrete) with $C$ given by

$$
C(d, a)=\sum_{i} d_{i} a_{i}, \quad(d, a) \in \Delta(Y)
$$

is a discrete global convexity associated with $\mathscr{A}=2^{Y}, \operatorname{conv}(A)=\operatorname{co}(A), A \subset Y$.

Proof. Condition (E) is trivially satisfied, because the uniform base $\mathscr{B}$ of $Y$ consists of only one element $B_{0}=\{(b, b): b \in Y\}$. Now we observe that $C(\cdot, a): \Delta_{n} \rightarrow Z$ is continuous as a linear map on a finite-dimensional space, so (D) of Definition 2.1 holds for $\mathscr{V}$ the class of all continuous maps.

5. Constructing a convexity on a topological space. Our goal is to construct a regular convexity on a given uniform space. Throughout this section we assume that the index set $I$ is well ordered. Let

$$
\begin{aligned}
& \mathscr{A}=\{\{y\}: y \in Y\}, \\
& Q=\cup_{y \in Y} \Delta(\{y\})=\left\{(d, a) \in \Delta(Y) \text { : for some } y \in Y, d_{i} \neq 0 \Longrightarrow a_{i}=y\right\} .
\end{aligned}
$$

LEMMA 5.1. Suppose $Y$ is Hausdorff compact and infinite. Let $\Delta^{\prime}(Y)$ be the quotient space of $\Delta(Y)$ with respect to the following equivalence relation:

$$
(d, a) \sim\left(d^{\prime}, a^{\prime}\right) \quad \text { if, for any } y \in Y, \sum_{a_{i}=y} d_{i}=\sum_{a_{i}^{\prime}=y} d_{i}^{\prime}
$$

Let $C: Q \rightarrow Y$ be given by $C(x)=y$ for any $x \in \Delta(\{y\})$. Then

(i) $\Delta^{\prime}(Y)$ is a Hausdorff uniform space and the quotient map $p: \Delta(Y) \rightarrow \Delta^{\prime}(Y)$ is uniformly continuous,

(ii) (a) in $Q$, equivalence classes are $\Delta(\{y\}), y \in Y$,

(b) $p(\Delta(\{y\}))=y, y \in Y$, and

(c) $p(Q)=Y$.

Proof. First, $\Delta(Y)$ is normal, so $\Delta^{\prime}(Y)$ is Hausdorff. Next, for any $(d, a) \in \Delta(Y)$, $y \in Y$, let $S_{y}(d, a)=\sum_{a_{i}=y} d_{i}$. Then the set

$$
R=\left\{\left((d, a),\left(d^{\prime}, a^{\prime}\right)\right): \text { for any } y \in Y, S_{y}(d, a)=S_{y}\left(d^{\prime}, a^{\prime}\right)\right\}
$$

determines the equivalence relation $\sim$. To ensure that $\Delta^{\prime}(Y)$ has a uniformity, we need to check that for any $W \in \mathscr{B}, \varepsilon>0, m \in \Omega$, there are $V \in \mathscr{B}, \delta>0, n \in \Omega$, such that

$$
V^{\delta, n}+R+V^{\delta, n} \subset R+W^{\varepsilon, m}+R
$$

which means that the equivalence relation $\sim$ and the uniformity of $\Delta(Y)$ are weakly compatible (see [18, page 24]), so (i) holds. We will show that $\Delta(Y) \times \Delta(Y) \subset R+W^{\varepsilon, m}+R$. 
Consider

$$
\begin{aligned}
R+W^{\varepsilon, m}+R=\{ & \left((d, a),\left(d^{\prime}, a^{\prime}\right)\right): \text { there is an }(r, b) \in \Delta(Y) \text { with } \\
& \left.((d, a),(r, b)) \in R,\left((r, b),\left(d^{\prime}, a^{\prime}\right)\right) \in W^{\varepsilon, m}+R\right\} \\
=\{ & \left((d, a),\left(d^{\prime}, a^{\prime}\right)\right): \\
& \exists(r, b) \in \Delta(Y) \text { with } S_{y}(d, a)=S_{y}(r, b), \forall y \in Y, \\
& \exists\left(r^{\prime}, b^{\prime}\right) \in \Delta(Y) \text { with } S_{y}\left(d^{\prime}, a^{\prime}\right)=S_{y}\left(r^{\prime}, b^{\prime}\right), \forall y \in Y, \\
& \text { and } \left.\left|r_{i}-r_{i}^{\prime}\right|<\varepsilon,\left(b_{i}, b_{i}^{\prime}\right) \in W, i \in m\right\} \\
=\{ & \left((d, a),\left(d^{\prime}, a^{\prime}\right)\right): \text { there are }(r, b),\left(r^{\prime}, b^{\prime}\right) \in \Delta(Y) \text { with } \\
& (1)\left|r_{i}-r_{i}^{\prime}\right|<\varepsilon,\left(b_{i}, b_{i}^{\prime}\right) \in W, i \in m, \\
& (2) S_{y}(d, a)=S_{y}(r, b), \forall y \in Y, \\
& \text { (3) } \left.S_{y}\left(d^{\prime}, a^{\prime}\right)=S_{y}\left(r^{\prime}, b^{\prime}\right), \forall y \in Y\right\} .
\end{aligned}
$$

Suppose $\left((d, a),\left(d^{\prime}, a^{\prime}\right)\right) \in \Delta(Y) \times \Delta(Y)$. Assume for simplicity that $m=\{0,1, \ldots, M\}$. Choose $z \in Y$ such that $d_{i} \neq 0 \Rightarrow z \neq a_{i}$ and $d_{i}^{\prime} \neq 0 \Rightarrow z \neq a_{i}^{\prime}$ for all $i \in I$. To get $(r, b),\left(r^{\prime}, b^{\prime}\right) \in \Delta(Y)$ as above, let

$$
r_{i}=r_{i}^{\prime}=0, \quad b_{i}=b_{i}^{\prime}=z, \quad i \in m=\{0,1, \ldots, M\} .
$$

Then condition (1) is satisfied. Now, let

$$
r_{M+i+1}=d_{i}, \quad r_{M+i+1}^{\prime}=d_{i}^{\prime}, \quad b_{M+i+1}=a_{i}, \quad b_{M+i+1}^{\prime}=a_{i}^{\prime}, \quad i=0,1, \ldots
$$

(it means that we obtain $(r, b)$ and $\left(r^{\prime}, b^{\prime}\right)$ by "shifting" coordinates of $(d, a)$ and $\left(d^{\prime}, a^{\prime}\right) M$ steps to the right). Then conditions (2) and (3) are also satisfied. Therefore we have

$$
R+W^{\varepsilon, m}+R=\Delta(Y) \times \Delta(Y)
$$

If $(d, a) \in Q$ and $d_{i} \neq 0$, then $a_{i}=y$ for all $i$ and some $y \in Y$, and $p(d, a)=y$. Then for any $V \in \mathscr{B}, n \in \Omega, \varepsilon>0$, we have

$$
\begin{gathered}
B^{*}\left((d, a), V^{\varepsilon, n}\right) \cap Q=\left\{\left(d^{\prime}, a^{\prime}\right) \in \Delta(Y): \text { for some } y^{\prime} \in B(y, V),\right. \\
\left.d_{i}^{\prime} \neq 0 \Longrightarrow a_{i}^{\prime}=y^{\prime}, \text { and }\left|d_{i}-d_{i}^{\prime}\right|<\varepsilon, i \in n\right\} \\
=\cup_{y^{\prime} \in B(y, V)}\left\{\left(d^{\prime}, a^{\prime}\right) \in \Delta(Y): d_{i}^{\prime} \neq 0 \Longrightarrow a_{i}^{\prime}=y^{\prime},\right. \\
\left.\left|d_{i}-d_{i}^{\prime}\right|<\varepsilon, i \in n\right\} .
\end{gathered}
$$

Therefore we have

$$
p\left(B^{*}\left((d, a), V^{\varepsilon, n}\right) \cap Q\right)=B(y, V),
$$

and we conclude that $p(Q)=Y$.

THEOREM 5.2. Let $Y$ be a Hausdorff compact ANR and let $\mathscr{V}$ be the set of all u.s.c. multifunctions with values either singletons or $Y$. Then there exists a convexity $\kappa=$ $(Y, C, Y)$ associated with $\mathscr{A}=\{\{y\}: y \in Y\}, \operatorname{conv}(\{y\})=\{y\}$ for all $y \in Y$. 
Proof. By Lemma 5.1, $\Delta^{\prime}(Y)$ is Hausdorff and $p(Q)=Y$ is compact. Hence $p(Q)$ is closed in $\Delta^{\prime}(Y)$. Then, there exists an open neighborhood $N_{V} \subset \Delta^{\prime}(Y)$ of $p(Q)$ and a continuous function $C: \overline{N_{V}} \rightarrow Y$ that extends $I d_{Y}$ and $C$ is uniformly continuous. Now if we extend $C$ on the whole $\Delta^{\prime}(Y)$ by putting $C(d, a)=Y$ for $(d, a) \notin N_{V}$, then $C$ is uniformly u.s.c. (since $N_{V}$ is open). It is routine to check that $C$ satisfies conditions $(\beta)-(\delta)$.

6. Preliminaries from general topology. Presenting necessary definitions we mostly follow Engelking [6].

DefinITION 6.1. The weight $w(Y)$ is the cardinality of $\mathscr{B}$. Let $\varphi(Y)$ be the largest cardinal number $\mu \leq \omega$ such that the intersection of any family of elements of $\mathscr{B}$ whose cardinality is less then $\mu$ contains an element of $\mathscr{B}$.

Note that if $\mathscr{\beta}=\left\{U_{\beta}: \beta<\mu\right\}$, where $\mu$ is an infinite ordinal, is ordered by inclusion (in this case $Y$ is called a $\mu$-metrizable space [13]), then $\varphi(Y)=w(Y)^{+}$, where $\alpha^{+}$stands for the least cardinal number larger than $\alpha$.

DEFINITION 6.2. For a topological space $X$, the Lindelof number $l(X)$ is the least cardinal number $\lambda$ such that every open cover of $X$ has a subcover whose cardinality is less than $\lambda$ ("at most" in [6]). Let $l^{\prime}(X)$ be the largest cardinal number $\mu \leq \omega$ such that any open cover of $X$ whose cardinality is less than $\mu$ has a finite subcover. Let $p(X)$ be the largest cardinal number $\kappa \leq \omega$ such that any open cover of $X$ whose cardinality is less than $\kappa$ has a locally finite open refinement.

Then $X$ is known [27] as finally $\lambda$-compact (or $\lambda$-Lindelöf [13]) and initially $\mu$-compact, respectively.

DEFINITION 6.3. For the uniform space $Y$, let $l_{u}(Y)$ be the least cardinal number $\lambda$ such that for every $V \in \mathscr{B}$, the cover $\{B(x, V): x \in Y\}$ has a subcover whose cardinality is less than $\lambda$.

The proof of the following proposition is similar to the one of [6, Theorem 3.1.23].

Proposition 6.4. Suppose $\mathscr{B}=\left\{U_{i}: i \in J\right\}$ is partially ordered by inclusion and $\varphi(Y) \geq l(Y)$. Then any net $\left\{y_{i}: i \in J\right\}$ in $Y$ has a convergent subnet.

7. The main selection theorems. Since Theorems 1.1, 1.2, 1.3, and 1.4 deal with two types of multifunctions (u.s.c. and l.s.c.), we shall consider maps $T: X \rightarrow Y$ and $R: Z \rightarrow X$ of either kind between the two spaces $(Z=Y)$ and a fixed point of their composition $T \circ R: Y \rightarrow Y$.

If a multifunction $T: X \rightarrow Y$ has admissible images, then its convex hull $\operatorname{conv}(T)$ : $X \rightarrow Y$ is given by

$$
\operatorname{conv}(T)(x)=\operatorname{conv}(T(x)), \quad x \in X
$$

Michael proves his selection theorem for his convex structures [21] by considering a sequence of "almost continuous" selections, while for locally convex topological vector spaces he constructs [20] a sequence of continuous "almost selections." The former 
yields sharper selection results, the latter requires an additional restriction on the convexity (some neighborhood of an admissible set is admissible, as in Proposition 12.8), but allows us to proceed directly to fixed point theorems.

THEOREM 7.1 (almost selection theorem). Let $X$ be a normal topological space, $\kappa=$ $(Y, C, Z)$ a convexity, $Y^{\prime}$ a subset of $Y$. Suppose also that

(i) $T: X \rightarrow Y$ is admissible-valued l.s.c. and $T(x) \cap Y^{\prime} \neq \varnothing$ for any $x \in X$,

(ii) $p(X) \geq l_{u}\left(Y^{\prime}\right)$.

Then for any $U \in \mathscr{B}$, there exist $a \in\left(Y^{\prime}\right)^{\omega}$ and a continuous function $f: X \rightarrow \Delta_{\omega}$ satisfying the following conditions:

(a) for any $x \in X$, there is an open neighborhood $G$ of $x$ such that $f(G) \subset \Delta_{n} \subset \Delta_{\omega}$ for some $n \geq 0$,

(b) $f(X) \times\{a\} \subset Q^{\prime}$, and

(c) $C(f(x), a) \subset B(\operatorname{conv}(T)(x), U)$ for all $x \in X$.

If, in addition, $Y^{\prime}$ is admissible, then

$$
C(f(x), a) \subset \overline{\operatorname{conv}}\left(Y^{\prime}\right) .
$$

If, moreover, (ii') $l^{\prime}(X) \geq l_{u}\left(Y^{\prime}\right)$, then we have $\left(\mathrm{a}^{\prime}\right) f(X) \subset \Delta_{n} \subset \Delta_{\omega}$ for some $n \geq 0$.

Proof. Let $U \in \mathscr{B}$. Then condition(E) reads as follows: there exists $W \in \mathscr{B}$ such that

$$
C(\Delta(B(A, W))) \subset B(\operatorname{conv}(A), U) \text { for all admissible } A \subset Y .
$$

Let $M=\left\{B(y, W): y \in Y^{\prime}\right\}$. By definition of $l_{u}\left(Y^{\prime}\right), M$ has a subcover $M^{\prime}$ with $\left|M^{\prime}\right|<$ $l_{u}\left(Y^{\prime}\right)$. But $T: X \rightarrow Y$ is l.s.c., so $N=\left\{T^{-1}(G): G \in M^{\prime}\right\}$ consists of open sets. Moreover, if $x \in X$, then $T(x) \cap G \neq \varnothing$ for some $G \in M^{\prime}$. Hence $x$ belongs to $T^{-1}(G)$, so $N$ is an open cover of $X$. By (ii), we have $|N|=\left|M^{\prime}\right|<l_{u}\left(Y^{\prime}\right) \leq p(X)$. Therefore, by definition of $p(X), N$ has a locally finite open refinement $N^{\prime}$. Since $|I|=\omega>2^{|X|}$, we can assume that $N^{\prime}=\left\{Q_{k}: k \in I\right\}\left(Q_{k}=\varnothing\right.$ for some $\left.k \in I\right)$. From the definitions of $M, N, N^{\prime}$, it follows that for all $k \in I, Q_{k} \subset T^{-1}\left(G_{k}\right)$, where $G_{k}=B\left(a_{k}, W\right)$ for some $a_{k} \in Y^{\prime}$ (here we assign an index to $G_{k}$ and $a_{k}$ according to this inclusion). Then let $a=\left(a_{i}\right)_{i \in I} \in\left(Y^{\prime}\right)^{\omega}$. From the fact that $X$ is normal and Michael's lemma it follows that there exists a partition of unity subordinate to $N^{\prime}$, i.e., there are continuous functions $f_{k}: X \rightarrow[0,1], k \in I$, satisfying

$$
\begin{gathered}
f_{k}(x)=0 \text { for any } x \notin Q_{k}, k \in I, \\
\sum_{k \in I} f_{k}(x)=1 \text { for any } x \in X .
\end{gathered}
$$

Now let

$$
f(x)=\sum_{k \in I} f_{k}(x) e_{k},
$$

where $e_{k}, k \in I$, are the vertices of $\Delta_{\omega}$. Then $f: X \rightarrow \Delta_{\omega}$ is a continuous function. Since $N^{\prime}$ is locally finite, for each $x \in X$, there are a neighborhood $G$ of $x$ and a finite set $S \subset I$ such that $\left.f_{k}\right|_{G}$ is nonzero only for $k \in S$. Therefore we have $f(G) \subset \Delta_{S}$, so (a) is satisfied. Moreover, if (ii') holds, then $N^{\prime}$ is finite and so $\left(\mathrm{a}^{\prime}\right)$ holds. 
Take $x \in X$. Let

$$
K=\left\{k \in I: T(x) \cap G_{k} \neq \varnothing\right\} \quad \text { and } \quad A_{K}=\left\{a_{k}: k \in K\right\} \subset Y^{\prime} .
$$

If $k \in I$ is such that $f_{k}(x) \neq 0$ then $x \in Q_{k} \subset T^{-1}\left(G_{k}\right)$, or $T(x) \cap G_{k} \neq \varnothing$. Hence $k \in K$. By definition of $f$, this implies that

$$
f(x)=\sum_{k \in I} f_{k}(x) e_{k}=\sum_{k \in K} f_{k}(x) e_{k} \in \Delta_{K},
$$

where $e_{k}, k \in I$, are the unit vectors of $[0,1]^{\omega}$. Next, consider

$$
\Delta_{K} \times\{a\}=\left\{(d, a): d_{i} \neq 0 \Longrightarrow a_{i} \in A_{K}\right\} \subset \Delta\left(A_{K}\right) .
$$

By definition of $K$, we have $a_{k} \in B(T(x), W)$ for all $k \in K$, or

$$
A_{K} \subset B(T(x), W) \cap Y^{\prime} .
$$

From (7.7), (7.8), and (7.9) it follows that

$$
f(x) \times\{a\} \in \Delta_{K} \times\{a\} \subset \Delta\left(A_{K}\right) \subset \Delta\left(B(T(x), W) \cap Y^{\prime}\right) .
$$

Since $T(x)$ is admissible, $\Delta(B(T(x), W)) \subset Q^{\prime}$. Hence by (7.10), we have $f(X) \times\{a\} \subset$ $Q^{\prime}$ and, therefore, $C(f(x), a)$ is well defined. Thus, from (7.10) and (7.3) we have (c).

To finish the proof, we notice that (7.10) implies that $f(x) \times\{a\} \in \Delta\left(Y^{\prime}\right)$. Therefore we have

$$
C(f(x), a) \subset C\left(\Delta\left(Y^{\prime}\right)\right) \subset \overline{\operatorname{conv}}\left(Y^{\prime}\right) .
$$

COROLLARY 7.2 (continuous almost selection theorem). Let $X$ be a normal topological space, $\kappa=(Y, C, Z)$ a continuous convexity, $Y^{\prime}$ a subset of $Y$. Suppose also that

(i) $T: X \rightarrow Y$ is l.s.c. with admissible images and $T(x) \cap Y^{\prime} \neq \varnothing$ for any $x \in X$,

(ii) $p(X) \geq l_{u}\left(Y^{\prime}\right)$.

Then for any $U \in \mathscr{B}$, there exists a continuous $V$-almost selection for the multifunction $\operatorname{conv}(T): X \rightarrow Z$, i.e., there is a continuous function $g: X \rightarrow Z$ such that

$$
g(x) \in B(\operatorname{conv}(T(x)), U) \quad \forall x \in X .
$$

If, moreover, $Y^{\prime}$ is admissible, then we have

$$
g(X) \subset \overline{\operatorname{conv}}\left(Y^{\prime}\right) .
$$

DEFINITION 7.3. We say that the convexity $\kappa=(Y, C, Z)$ has a convex uniform base $\mathscr{B}$ if

$$
y \in Y, \quad U \in \mathscr{B}, \quad D \in \mathscr{C} \Longrightarrow B(y, U) \cap D \in \mathscr{C} .
$$

THEOREM 7.4 (continuous selection theorem). Let $X$ be a normal topological space, $Y$ be a complete uniform space, $\kappa=(Y, C, Z)$ a continuous convexity with a countable convex uniform base $\mathscr{B}$, and suppose that the uniform topology of $Y$ is finer than the 
topology of Z. Suppose also that

(i) $T: X \rightarrow Y$ is l.s.c. with nonempty convex images,

(ii) $p(X) \geq l_{u}(Y)$.

Then the multifunction $\bar{T}: X \rightarrow Z$ has a continuous selection, that is, there is a continuous map $g: X \rightarrow Z$ such that

$$
g(x) \in \overline{T(x)} \quad \forall x \in X \text { (closure in } Y \text { ). }
$$

Proof. Let $\mathscr{B}=\left\{U_{1}, U_{2}, \ldots\right\}$. Without loss of generality, we can assume that

$$
2 U_{n+1} \subset U_{n}, \quad n=1,2, \ldots
$$

Then according to Corollary 7.2 (with $Y^{\prime}=Y$ ), for any nonempty convex-valued l.s.c. map $G: X \rightarrow Y$, for any $U \in \mathscr{B}$, there is a continuous $g: X \rightarrow Z$ with

$$
g(x) \in B(G(x), U) \quad \forall x \in X .
$$

We inductively construct a sequence of continuous functions $g_{n}: X \rightarrow Z, n=1,2, \ldots$, such that

$$
\begin{aligned}
& g_{n}(x) \in B\left(T(x), U_{n+1}\right) \quad \forall x \in X, n=1,2, \ldots, \\
& g_{n}(x) \in B\left(g_{n-1}(x), U_{n-1}\right) \quad \forall x \in X, n=2,3, \ldots
\end{aligned}
$$

By (7.17), there is a $g_{1}$ so that (7.18) holds for $n=1$. Assume that we have constructed $g_{1}, \ldots, g_{n-1}$ satisfying these conditions. Then let

$$
G(x)=B\left(g_{n-1}(x), U_{n}\right) \cap T(x) .
$$

Then $G$ is l.s.c. By (7.18), for $n-1$, we have $g_{n-1}(x) \in B\left(T(x), U_{n}\right)$, so $G(x)$ is nonempty, and it is convex because the base is convex. Therefore by (7.17), there is a continuous map $g_{n}: X \rightarrow Z$ with

$$
g_{n}(x) \in B\left(G(x), U_{n+1}\right) \quad \forall x \in X .
$$

Then there is a $y \in G(x)$ such that

$$
\left(g_{n}(x), y\right) \in U_{n+1} .
$$

By (7.20), we have $G(x) \subset T(x)$, so (7.21) implies (7.18). From (7.20), it also follows that $G(x) \subset B\left(g_{n-1}(x), U_{n}\right)$. Therefore we have $\left(y, g_{n-1}(x)\right) \in U_{n}$, and now, from (7.22) and (7.16), it follows that $\left(g_{n}(x), g_{n-1}(x)\right) \in U_{n-1}$. Hence (7.19) holds. Thus, we have constructed a sequence $\left\{g_{n}: n=1,2, \ldots\right\}$ satisfying the required conditions.

Now (7.16) implies that this is a Cauchy sequence. Therefore $\left\{g_{n}: n=1,2, \ldots\right\}$ converges to a map $g: X \rightarrow Z$. And from (7.18), it follows that $g(x) \in \overline{T(x)}$ for all $x \in X$. To finish the proof we observe that $g: X \rightarrow Z$ is the uniform limit of $\left\{g_{n}\right\}$ with respect to the uniformity of $Y$. Therefore $g$ is continuous, since $Y$ is finer than $Z$. 


\section{More selection theorems}

THEOREM 8.1 (Michael-type selection theorem). Let $X$ be a (Hausdorff) paracompact space, $\kappa=(Y, C, Y)$ a continuous convexity, $Y$ complete with a countable convex uniform base, $T: X \rightarrow Y$ l.s.c. with nonempty convex images. Then $\bar{T}$ has a continuous selection.

Proof. In Theorem 7.4, we let $Z=Y$ and notice that $p(X)=\omega \geq l_{u}(Y)$, so condition (ii) of the theorem holds.

This theorem implies the following results: (1) the Michael selection Theorem 1.3 for Banach spaces, (2) Theorem 1.3 of Michael [21] for Michael's convex structures (with two additional assumptions: (a) for any $x \in X$, any $W \in \mathscr{B}, B(\varphi(x), W)$ is $M$ admissible, (b) for any $y \in E$, any $W \in \mathscr{B}, B(y, W)$ is $M$-convex), (3) Theorem 3.3 of Horvath [15] for $H$-spaces, (4) part (2) of Theorem 3.5 of van de Vel [30, page 440] (or part (b) of Theorem 4.3 [29]). (Here (3) and (4) may be looked at as selection theorems for an l.s.c. map with convex range.)

An example of a pair $X, Y$ that satisfies conditions (ii) of Theorem 7.4 but is not covered by the Michael selection theorem: $X$ is normal but not necessarily paracompact and $Y$ is paracompact. Another example: $X$ is countably paracompact, or countably compact, such as the space $W$ of all countable ordinal numbers, and $Y$ is a separable Banach space, such as $l_{2}$ or $C[0,1]$. Nedev [22] proved a Michael-type selection theorem for $X=W$ and $Y$ a reflexive Banach space.

THEOREM 8.2 (Browder-type selection theorem). Let $X$ be a (Hausdorff) paracompact space, and suppose $(Y, C, Z)$ is a discrete convexity, and $T: X \rightarrow Y$ has nonempty admissible images and open fibers. Then $\operatorname{conv}(T): X \rightarrow Z$ has a continuous selection.

Proof. In Corollary 7.2 we let $Y$ be discrete and $Y^{\prime}=Y$. Then $T: X \rightarrow Y$ is l.s.c., so condition (i) of the theorem holds. But since $p(X)=\omega \geq l_{u}(Y)$, (ii) also holds. Finally, we notice that an almost selection with respect to the discrete topology is in fact a selection.

This corollary implies the following results: (1) the Browder selection Theorem 1.4 for topological vector spaces, (2) Theorem 3.2 of Horvath [15] for $H$-spaces, (3) van de Vel's version of the Browder selection theorem [30, page 450].

9. Classes of maps with fixed point conditions. Motivated by Ben-El-Mechaiekh and Deguire [2], Park and Kim [24] define an abstract class $u_{c}^{\kappa}(Y, X)$ of maps that helps reduce the fixed point problem to the one for multifunctions on simplexes.

Examples of $u_{c}^{\kappa}(Y, X)$ are, for instance, the classes of all u.s.c. multifunctions with compact convex values in locally convex topological vector spaces, acyclic maps, and approachable maps. Taking this one step further, we introduce the following.

Definition 9.1. Let $X$ be a topological space, $\kappa=(Y, C, Z)$ be a convexity and $Y^{\prime}$ a subset of $Y$ (or $Z$ ). Then the class $\mathscr{F}_{\kappa}\left(Y^{\prime}, X\right)$ is defined as the class of all multifunctions $F: Z \supset Y^{\prime} \rightarrow X$ such that for any simplex $\Delta_{n} \subset \Delta_{\omega}$, any $a \in\left(Y^{\prime}\right)^{\omega}$, and any continuous function $f: X \rightarrow \Delta_{n}$, the multifunction 


$$
\left.f \circ F \circ C\right|_{\Delta_{n} \times\{a\}}: \Delta_{n} \rightarrow \Delta_{n}
$$

has a fixed point.

By Definitions 2.1 and 4.1, $C(\cdot, a): \Delta_{n} \rightarrow Z, V \in B$, is continuous when $\kappa=(Y, C, Z)$ is a continuous convexity. Therefore the Brouwer fixed point theorem implies the following proposition.

Proposition 9.2. If $\kappa=(Y, C, Z)$ is a continuous global convexity, then $\mathscr{F}_{\kappa}(Y, X)$ contains all continuous functions $\psi: Z \rightarrow X$.

If $\mathscr{V}$ is the set of all acyclic maps, then we say that a convexity associated with $\mathscr{V}$ is acyclic. Then Theorem 1.6 implies the following proposition.

Proposition 9.3. If $\kappa=(Y, C, Z)$ is a global acyclic convexity, then $\mathscr{F}_{\kappa}(Y, X)$ contains all admissible in the sense of Gorniewicz (and, therefore, all acyclic) multifunctions $F: Z \rightarrow X$.

Proposition 9.4. If $\kappa=(Y, C, Y)$ is a global acyclic convexity and $Y^{\prime}$ is a closed convex subset of $Y$, then $\mathscr{F}_{K}\left(Y^{\prime}, X\right)$ contains all u.s.c. multifunctions $F: Y^{\prime} \rightarrow X$ such that $F(x)$ is compact and acyclic for all $x \in Y^{\prime}$.

Proposition 9.5. If $\kappa=(Y, C, Y)$ is a global continuous convexity, then we have

$$
u_{c}^{\kappa}(Y, X) \subset \mathscr{F}_{\kappa}(Y, X) .
$$

\section{The main fixed point theorems}

THEOrem 10.1 (almost fixed point theorem). Let $X$ be a normal topological space, $\kappa=(Y, C, Z)$ a global convexity. Suppose also that the following holds:

(i) $R \in \mathscr{F}_{\kappa}(Y, X)$,

(ii) $T: X \rightarrow Y$ is admissible-valued l.s.c.,

(iii) $l^{\prime}(X) \geq l_{u}(Y)$.

Then the multifunction $\operatorname{conv}(T) \circ R: Y \rightarrow Y$ has a $U$-almost fixed point for any $U \in \mathscr{B}$, i.e., there is a

$$
y \in B(\operatorname{conv}(T) \circ R(y), U) .
$$

Proof. By Theorem 7.1, with (ii'), for any $U \in \mathscr{B}$ there exist $a \in Y^{\omega}$ and a continuous function $f: X \rightarrow \Delta_{J}$ with $J$ finite such that $C(f(x), a) \subset B(\operatorname{conv}(T)(x), U)$ for all $x \in X$. Since the convexity is global, we can define a map $\Psi: \Delta_{J} \rightarrow Y$ by $\Psi=\left.C\right|_{\Delta_{J} \times\{a\}}$. Since $R \in \mathscr{F}_{K}(Y, X)$, the multifunction $F=f \circ R \circ \Psi: \Delta_{J} \rightarrow \Delta_{J}$ has a fixed point, that is, there exists a $d \in f(R(\Psi(d)))$. Therefore, there exist such $x \in X$ and $y \in Y$ that $y \in \Psi(f(x))$ and $x \in R(y)$. Hence

$$
y \in C(f(x), a) \subset B(\operatorname{conv}(T)(x), U) \subset B(\operatorname{conv}(T) \circ R(y), U) .
$$

TheOREM 10.2 (fixed point theorem). Let $X$ be a normal topological space, $\kappa=$ $(Y, C, Z)$ a global convexity. Suppose that the following conditions are satisfied: 
(i) $R \in \mathscr{F}_{K}(Y, X)$,

(ii) $T: X \rightarrow Y$ is admissible-valued l.s.c.,

(iii) $S: Y \rightarrow Y$ is closed-valued u.s.c. and $\operatorname{conv}(T) \circ R \subset S$,

(iv) $\varphi(Y) \geq l(Y), l^{\prime}(X) \geq l_{u}(Y)$.

Then $S$ has a fixed point.

Proof. Suppose $\mathscr{B}=\left\{U_{i}: i \in J\right\}$. By Theorem 10.1, for any $i \in J$, there is a $y_{i} \in$ $B\left(\operatorname{conv}(T) \circ R\left(y_{i}\right), U_{i}\right)$. Now we need to show that $y^{*} \in S\left(y^{*}\right)$ for some $y^{*} \in Y$. By Proposition 6.4, the net $\left\{y_{i}: i \in J\right\}$ has a convergent subnet. Therefore, we can simply assume that $y_{i} \rightarrow y^{*} \in Y$. Then for any $i \in J$, there exists a $k(i) \in J$ such that $y_{k} \in B\left(y^{*}, U_{i}\right)$ for all $k>k(i)$, or

$$
y^{*} \in B\left(y_{k}, U_{i}\right) \quad \forall k>k(i) .
$$

By (iii), $y_{k} \in B\left(S\left(y_{k}\right), U_{k}\right)$ for all $k \in J$. Therefore

$$
y_{k} \in B\left(S\left(y_{k}\right), U_{i}\right) \quad \forall k>i .
$$

Since $y_{i} \rightarrow y^{*}$ and the multifunction $S$ is u.s.c., then for any $i \in J$, there exists a $j(i) \in J$ such that

$$
S\left(y_{k}\right) \subset B\left(S\left(y^{*}\right), U_{i}\right) \quad \forall k>j(i) .
$$

Now using (10.3), (10.4), and (10.5), we obtain

$$
y^{*} \in B\left(S\left(y^{*}\right), 3 U_{i}\right) \quad \forall i \in J .
$$

Hence $y^{*} \in \overline{S\left(y^{*}\right)}=S\left(y^{*}\right)$, and the proof is completed.

11. More fixed point theorems. One can obtain Kakutani and Browder type theorems for spaces with generalized convexity by repeating arguments that work for topological vector spaces (see $[15,16,23,25,28]$ ). But then the results apply only to convex-valued multifunctions. Therefore they are never stronger than the EilenbergMontgomery Theorem 1.7, which deals with acyclic-valued multifunctions. So we apply a version of the Eilenberg-Montgomery theorem, Theorem 1.6, or use $\mathscr{F}_{\kappa}(X, X)$ to obtain sharper results.

THEOREM 11.1 (Kakutani-type fixed point theorem). Let $X$ be a (Hausdorff) compact topological space, $\kappa=(Y, C, Y)$ a regular convexity, $f: X \rightarrow Y$ a (single-valued) continuous map and $R \in \mathscr{F}_{K}(Y, X)$ u.s.c. Then $\overline{f \circ R}$ has a fixed point.

Proof. In Theorem 10.2, we let $T=f, S=\overline{f \circ R}$. Then (i) and (ii) hold because the convexity is regular: $\operatorname{conv}(\{y\})=\{y\}$. Also, since $Y$ is compact, we have $l^{\prime}(X) \geq \aleph_{0}=$ $l(Y)$ and $\varphi(Y) \geq \aleph_{0}=l(Y)$, so (iv) holds.

This theorem implies the following results: (1) the Kakutani Theorem 1.1 for locally convex topological vector spaces, (2) Corollary to Theorem 6 of Horvath [16] for l.c. spaces, (3) Theorem 6.15 of van de Vel [30, page 498], (4) Theorem 1 of Hadžić [12] for $H$-spaces (with the assumption that all points are $H$-convex and that space is normal). 
Theorem 11.2 (Browder-type fixed point theorem). Let $X$ be compact, $(Y, C, Z)$ be a discrete global convexity. Suppose that

(i) $R \in \mathscr{F}_{K}(Y, X)$,

(ii) $T: X \rightarrow Y$ is a multifunction with admissible images and open fibers.

Then the multifunction $\operatorname{conv}(T) \circ R$ has a fixed point.

Proof. Notice that if $Y$ is a discrete uniform space, then a multifunction $T: X \rightarrow Y$ has open fibers if and only if it is l.s.c. Therefore conditions (i), (ii) of this theorem are exactly conditions (i), (ii) of Theorem 10.2. Condition (iii) follows from the fact that $S=\operatorname{conv}(T) \circ R: Y \rightarrow Y$ is u.s.c. with respect to the discrete topology. Also, since $X$ is compact and $Y$ is discrete, we have $l^{\prime}(X)=\omega \geq|Y|=l_{u}(Y)$ and $\varphi(Y)=\omega \geq|Y|^{+}=$ $l(Y)$, so (iv) holds.

This theorem implies the following results: (1) the Browder fixed point Theorem 1.2, (2) Theorem 7 of Browder [3], (3) Theorem 4.3 of Horvath [15] and Theorem 3.1 of Ding and Tarafdar [4] for $H$-spaces, (4) Browder-type fixed point theorem of van de Vel [30, Section IV.6.28, page 506].

We can generalize the definition of convexity by considering a system of "approximative" convexity multifunctions converging to $C$. As a result we can obtain the results of this paper for such spaces as AES [2], ANES [10], the "comb space," and admissible in the sense of Klee subsets of topological vector spaces [11].

12. Appendix: other definitions of generalized convexity. In this section, we provide (without proof) comparison of our definition of convexity with those due to Horvath, van de Vel, and Michael.

12.1. $H$-spaces. The following notion, originating from the work of Horvath [14, 15], is a generalization of the convex hull in a topological vector space.

DEFINITION 12.1. A pair $\left(Z,\left\{\Gamma_{A}\right\}\right)$ will be called an $H$-space, if $Z$ is a topological space and $\left\{\Gamma_{A}\right\}$ is a family of contractible subsets of $Z$ indexed by all finite subsets of $Z$ so that

$$
\Gamma_{A} \subset \Gamma_{B} \quad \text { whenever } A \subset B .
$$

( $\left(Z,\left\{\Gamma_{A}\right\}\right)$ is called a $c$-space [15].) A set $A \subset Z$ is called $H$-convex if $\Gamma_{D} \subset A$ for any finite $D \subset A$, and the $H$-convex hull of a set $A \subset Y$ is given by

$$
\operatorname{conv}^{*}(A)=\cup\left\{\Gamma_{D}: D \subset A, D \text { is finite }\right\} .
$$

Proposition 12.2. Let $\left(Z,\left\{\Gamma_{A}\right\}\right)$ be an $H$-space. Then there is a global discrete convexity $(Y, C, Z)$ associated with some $C: \Delta(Y) \rightarrow Y, \mathscr{A}=2^{Y} \backslash\{\varnothing\}$, and conv given by: $\operatorname{conv}(A)=\operatorname{conv}^{*}(A), A \in \mathcal{A}$.

The next definition provides an analogue of local convexity.

DEFINITION 12.3. (1) (Horvath [15].) We say that an $H$-space $\left(Y,\left\{\Gamma_{A}\right\}\right)$ is an l.c. space if there is a uniform base $\mathscr{B}$ such as for any $U \in \mathscr{B}$,

$$
B(A, U) \text { is an } H \text {-convex set whenever } A \subset Y \text { is } H \text {-convex. }
$$


A metric space $(Y, d)$ is called a metric l.c. space if it is a $c$-space and $\forall \varepsilon>0,\{y \in Y$ : $d(y, E)<\varepsilon\}$ is an $H$-convex set if $E$ is an $H$-convex set, and open balls are $H$-convex.

(2) (Hadžić [12].) We say that an $H$-space $\left(Y,\left\{\Gamma_{A}\right\}\right)$ is of generalized Zima type if there is a uniform base $\mathscr{B}$ such that for every $U \in \mathscr{B}$, there exists a $V \in \mathscr{B}$ such that for every finite subset $D$ of $Y$ and every $H$-convex subset $A$ of $Y$ the following holds:

$$
A \cap B(z, V) \neq \varnothing \text { for every } z \in D \Longrightarrow A \cap B(u, U) \neq \varnothing \text { for every } u \in \Gamma_{D} .
$$

Proposition 12.4. Suppose that

(1) $\left(Y,\left\{\Gamma_{A}\right\}\right)$ is an l.c. space, or

(2) $\left(Y,\left\{\Gamma_{A}\right\}\right)$ is an $H$-space of generalized Zima type with $H$-convex points.

Then there is a regular convexity $(Y, C, Y)$ associated with $\mathscr{b}=\{H$-convex sets $\}$.

\section{2. van de Vel's uniform convex structures}

DEFINITION 12.5 [30, pages 3 and 304]. A pair $(Y, \mathscr{b})$, where $\mathscr{b}$ is a family of subsets of $Y$, called convex sets, is called a uniform convex structure if

(1) the empty set $\varnothing$ and the universal set $Y$ are in $\mathscr{C}$,

(2) $\mathscr{C}$ is stable for intersections, that is, if $\mathscr{D} \subset \mathscr{C}$ is nonempty, then $\cap \mathscr{D}$ is in $\mathscr{C}$,

(3) $\mathscr{C}$ is stable for nested unions, that is, if $\mathscr{D} \subset \mathscr{C}$ is nonempty and totally ordered by inclusion, then $\cup \mathscr{D}$ is in $\mathscr{C}$,

(4) there is a uniform base $\mathscr{B}$ such that for each $U \in \mathscr{B}$, there is a $V \in \mathscr{B}$ such that

$$
\text { for any } A \in \mathscr{C}, \operatorname{conv}(B(A, V)) \subset B(A, U) \text {, }
$$

where the convex hull conv is defined as:

$$
\operatorname{conv}(A)=\bigcap\{D \in \mathscr{C}: A \subset D\}, \quad A \subset Y .
$$

Proposition 12.6. If $(Y, \mathscr{b})$ is a uniform convex structure such that all elements of $\mathscr{C}$ are $A R$ 's, then there is a global continuous convexity $(Y, C, Y)$ associated with $\mathscr{C}$.

van de Vel says that his convex structure satisfies the $S_{4}$-axiom if two disjoint convex sets can be separated by two convex sets complement to each other. He calls convex hulls of finite sets polytopes. The statement below follows from Proposition 12.6 and van de Vel's selection theorem [30, Theorem 3.17, page 446].

Proposition 12.7. Let $(Y, \mathscr{C})$ be a metrizable van de Vel uniform convex structure satisfying the $S_{4}$-axiom with compact polytopes such that $\mathscr{b}$ contains only connected sets. Then there is a global continuous convexity $(Y, C, Y)$ associated with $\mathscr{C}$.

\subsection{Michael convex structures}

Proposition 12.8. Let $\left\{\left(M_{n}, k_{n}\right)\right\}$ be a Michael's convex structure. Let

$$
\begin{gathered}
\mathscr{A}=\left\{A \subset Y: B(A, W)^{n+1} \subset M_{n} \forall n \geq 0, W \in \mathscr{B}\right\}, \\
\operatorname{conv}(A)=\left\{k_{n}(t, x): x \in A^{n+1}, t \in \Delta_{n}, n \geq 0\right\}, \quad A \in \mathscr{A},
\end{gathered}
$$

(notice that $A$ can be empty even if $M_{n}$ are not). Then $(Y, C, Y)$ is a continuous convexity if $C: Q^{\prime} \rightarrow Y$, where $Q^{\prime}=\cup_{A \in \mathscr{A}, W \in \mathscr{B}} \Delta(B(A, W)$ ) (cf., (c8)), is defined as follows: for any $(d, a) \in Q^{\prime}$, 


$$
C(d, a)=k_{n}(t, x),
$$

where

$$
\begin{gathered}
t=\left(d_{i_{0}}, \ldots, d_{i_{n}}\right) \quad \text { and } \quad x=\left(a_{i_{0}}, \ldots, a_{i_{n}}\right), \\
\left\{i_{k}: k=0, \ldots, n\right\}=\left\{i \in I: d_{i} \neq 0\right\}, \quad i_{0}<\cdots<i_{n},
\end{gathered}
$$

(we assume that the index set I is totally ordered).

Let us consider an example of a Michael convex structure: $Y=M_{0}=\{0,1\} \subset \mathbf{R}, M_{n}=$ $\varnothing$ for $n \geq 1, k_{0}(t, x)=x$ for all $x \in Y, t \in[0,1]$. Let $E=Y=\{0,1\}, Q_{0}(t, x)=x$ for all $x \in Y, t \in[0,1]$. Then it is obvious that $Q_{1}$ does not exist. More generally, we can equip an $m$-sphere $\mathbf{S}^{m}, m \geq 0$, with a non-trivial Michael convex structure by using its local Euclidean structure. These examples show that Michael convex structures are not generalized by convex structures due to Park and Kim [24] and Pasicki [25].

ACKNOWLEDgement. This paper is a condensed version of two chapters of my doctoral thesis "Fixed points and coincidences" written under the guidance of Professor M.-E. Hamstrom at the University of Illinois at Urbana-Champaign.

\section{REFERENCES}

[1] C. Bardaro and R. Ceppitelli, Some further generalizations of Knaster-KuratowskiMazurkiewicz theorem and minimax inequalities, J. Math. Anal. Appl. 132 (1988), no. 2, 484-490. MR 89e:90219. Zbl 667.49016.

[2] H. Ben-El-Mechaiekh and P. Deguire, Approachability and fixed points for nonconvex set-valued maps, J. Math. Anal. Appl. 170 (1992), no. 2, 477-500. MR 94a:54103. Zbl 762.54033.

[3] F. E. Browder, The fixed point theory of multi-valued mappings in topological vector spaces, Math. Ann. 177 (1968), 283-301. MR 37\#4679. Zbl 176.45204.

[4] X. P. Ding and E. Tarafdar, Some coincidence theorems and applications, Bull. Austral. Math. Soc. 50 (1994), no. 1, 73-80. MR 95g:54036. Zbl 814.54028.

[5] S. Eilenberg and D. Montgomery, Fixed point theorems for multi-valued transformations, Amer. J. Math. 68 (1946), 214-222. MR 8,51a. Zbl 060.40203.

[6] R. Engelking, General Topology, 2nd ed., Sigma Series in Pure Mathematics, vol. 6, Heldermann Verlag, Berlin, 1989, Translated from the Polish by the author. MR 91c:54001. Zbl 684.54001.

[7] K. Fan, Fixed-point and minimax theorems in locally convex topological linear spaces, Proc. Nat. Acad. Sci. U.S.A. 38 (1952), 121-126. MR 13,858d. Zbl 047.35103.

[8] I. L. Glicksberg, A further generalization of the Kakutani fixed theorem, with application to Nash equilibrium points, Proc. Amer. Math. Soc. 3 (1952), 170-174. MR 13,764g. Zbl 046.12103.

[9] L. Gorniewicz, Homological methods in fixed-point theory of multi-valued maps, Dissertationes Math. (Rozprawy Mat.) 129 (1976), 71 pp. MR 52\#15438. Zbl 324.55002.

[10] L. Gorniewicz and A. Granas, Topology of morphisms and fixed point problems for set-valued maps, Fixed Point Theory and Applications (Marseille, 1989), Pitman Res. Notes Math. Ser., 252, Longman Sci. Tech., Harlow, 1991, pp. 173-191. MR 92f:54016. Zbl 760.54030.

[11] O. Hadžić, Fixed Point Theory in Topological Vector Spaces, Univerzitet u Novom Sadu, Institut za Matematiku, Novi Sad, 1984. MR 87m:47127. Zbl 576.47030.

[12] _ Almost fixed point and best approximations theorems in $H$-spaces, Bull. Austral. Math. Soc. 53 (1996), no. 3, 447-454. MR 97j:47080. Zbl 884.47035. 
[13] M. J. Harris, $\kappa$-Lindelöf $\kappa$-stratifiable spaces are $\kappa$-metrizable, Topology Appl. 73 (1996), no. 3, 295-298. MR 97i:54031. Zbl 859.54019.

[14] C. D. Horvath, Some results on multivalued mappings and inequalities without convexity, Nonlinear and Convex Analysis (Santa Barbara, Calif., 1985), Lecture Notes in Pure and Appl. Math., 107, Dekker, New York, 1987, pp. 99-106. MR 88g:47109. Zbl 619.55002.

[15] _ Contractibility and generalized convexity, J. Math. Anal. Appl. 156 (1991), no. 2, 341-357. MR 92f:52002. Zbl 733.54011.

[16] _ Extension and selection theorems in topological spaces with a generalized convexity structure, Ann. Fac. Sci. Toulouse Math. (6) 2 (1993), no. 2, 253-269. MR 94i:54043. Zbl 799.54013.

[17] T. Husain and E. Tarafdar, A selection and a fixed point theorem and an equilibrium point of an abstract economy, Int. J. Math. Math. Sci. 18 (1995), no. 1, 179-184. MR 95m:90026. Zbl 819.90015.

[18] I. M. James, Introduction to Uniform Spaces, London Mathematical Society Lecture Note Series, vol. 144, Cambridge University Press, Cambridge, 1990. MR 91m:54033. Zbl 719.54036.

[19] S. Kakutani, A generalization of Brouwer's fixed point theorem, Duke Math. J. 8 (1941), 457-459. MR 3,60c. Zbl 061.40304.

[20] E. Michael, Continuous selections. I, Ann. of Math. (2) 63 (1956), 361-382. MR 17,990e. Zbl 071.15902.

[21]__ Convex structures and continuous selections, Canad. J. Math. 11 (1959), 556-575. MR 22\#230. Zbl 093.36603.

[22] S. Y. Nedev, A selection example, C. R. Acad. Bulgare Sci. 40 (1987), no. 11, 13-14. MR 89e:54034. Zbl 632.54014.

[23] S. Park, Foundations of the KKM theory via coincidences of composites of upper semicontinuous maps, J. Korean Math. Soc. 31 (1994), no. 3, 493-519. MR 95i:47104. Zbl 829.49002.

[24] S. Park and H. Kim, Coincidence theorems for admissible multifunctions on generalized convex spaces, J. Math. Anal. Appl. 197 (1996), no. 1, 173-187. MR 97b:47072. Zbl 851.54039.

[25] L. Pasicki, A fixed point theory and some other applications of weeds, Opuscula Math. (1990), no. 7, 96 pp. MR 92h:54061. Zbl 709.54024.

[26] P. Saveliev, A Lefschetz-type coincidence theorem, Fund. Math. 162 (1999), no. 1, 65-89. CMP 1734 818. Zbl 934.55003.

[27] R. M. Stephenson, Jr., Initially $\kappa$-compact and related spaces, Handbook of SetTheoretic Topology (Amsterdam, New York), North-Holland, 1984, pp. 603-632. MR 86i:54024. Zbl 588.54025.

[28] E. Tarafdar, Fixed point theorems in locally $H$-convex uniform spaces, Nonlinear Anal., Theory Methods Appl. 29 (1997), no. 9, 971-978. MR 98c:54037. Zbl 904.47048.

[29] M. van de Vel, A selection theorem for topological convex structures, Trans. Amer. Math. Soc. 336 (1993), no. 2, 463-496. MR 93f:46011. Zbl 781.52002.

[30] M. L. J. van de Vel, Theory of Convex Structures, North-Holland Mathematical Library, vol. 50, North-Holland Publishing Co., Amsterdam, 1993. MR 95a:52002. Zbl 785.52001.

[31] E. Zeidler, Nonlinear Functional Analysis and its Applications. I, Fixed-point theorems. Translated from the German by Peter R. Wadsack, Springer-Verlag, New York, Berlin, 1986. MR 87f:47083. Zbl 794.47033.

Peter SAVeliev: Department of Mathematics, University of Illinois, 1409 West Green STREET, URBANA, IL 61801, USA

E-mail address: save1 i ev@math. uiuc. edu 


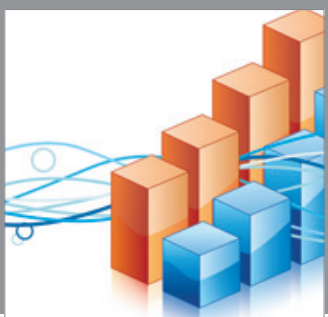

Advances in

Operations Research

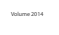

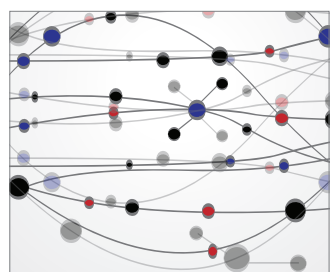

\section{The Scientific} World Journal
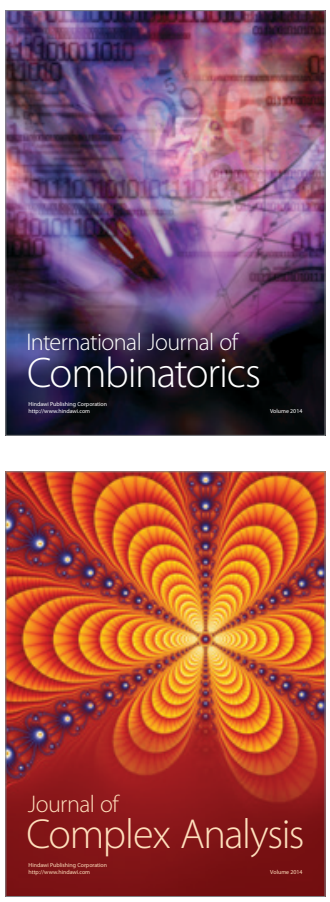

International Journal of

Mathematics and

Mathematical

Sciences
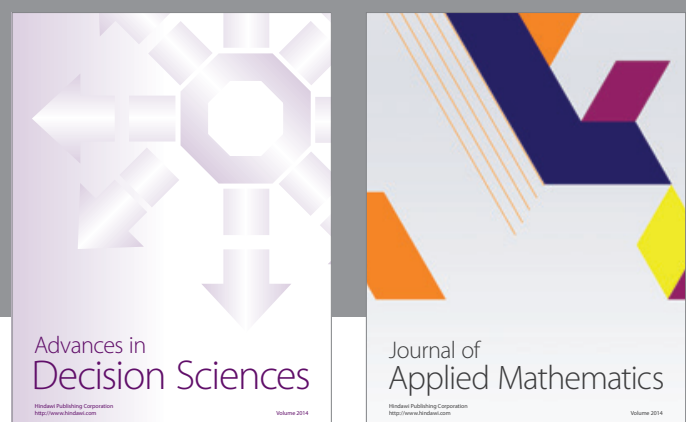

Journal of

Applied Mathematics
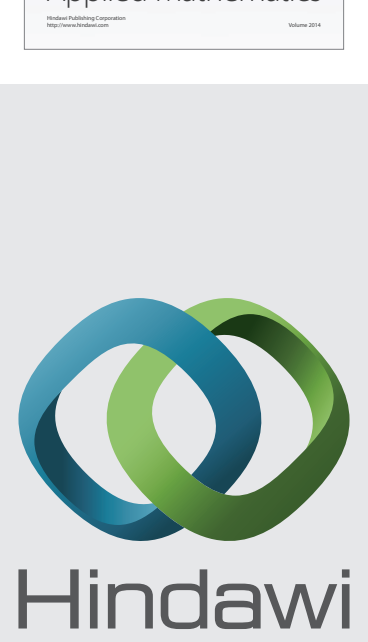

Submit your manuscripts at http://www.hindawi.com
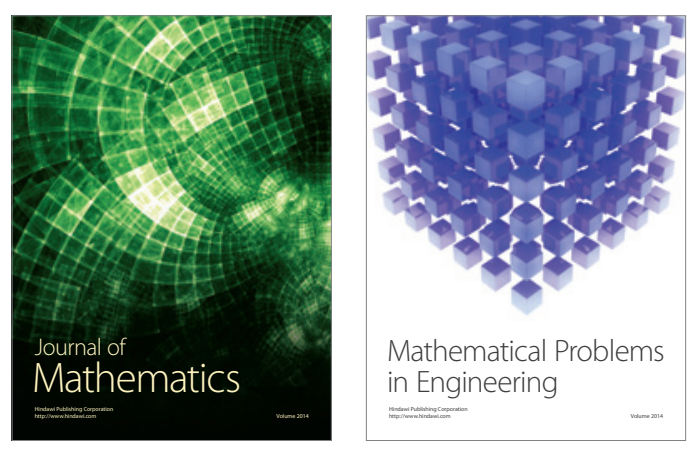

Mathematical Problems in Engineering
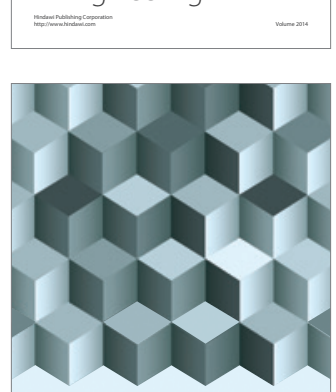

Journal of

Function Spaces
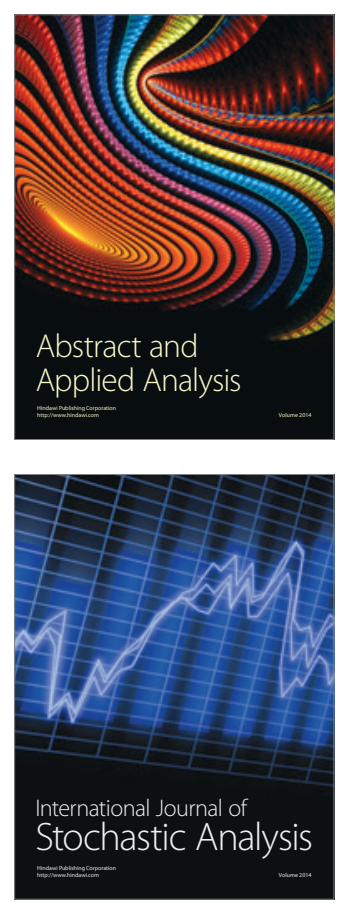

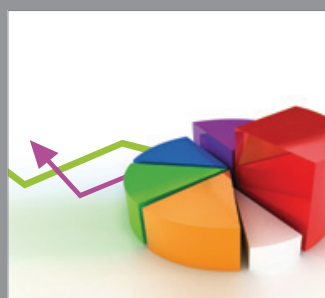

ournal of

Probability and Statistics

Promensencen
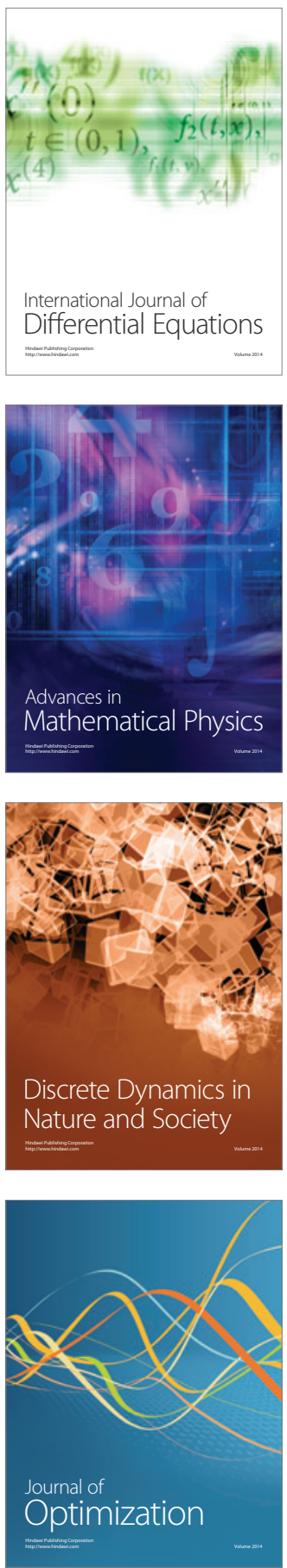